\title{
A Novel Technique for Link Recovery in Energy Efficient DRINA Protocol for Wireless Sensor Network
}

\author{
Mankirat Kaur ${ }^{1}$, Anjana Sharma ${ }^{2}$, Bhupinder Kaur ${ }^{3}$ \\ PG Student, ECE Department, CGC College of Engineering, Landran, Punjab ${ }^{1,3}$ \\ Assistant Professor, ECE Department, CGC College of Engineering, Landran, Punjab ${ }^{2}$
}

\begin{abstract}
The Wireless Sensor Network (WSN) is the type of Ad hoc network. WSN is the self configuring networks; any sensor node can join or leave the network when they want. In WSN the main issue is battery consumption so to conserve the network lifetime many protocols are designed. WSN's are deployed in the far places like forests, deserts etc so it is very difficult to recharge or replace the battery of the sensor nodes. In these conditions, we focus to reduce the battery consumption of the sensor nodes. In this paper, we are proposing a new technique on enhancement of DRINA protocol, to reduce battery consumption and recover link failure. It will be based on the static clustering using relay nodes. Before data transmission sensor nodes form the cluster dynamically using the neural network and weights are adjust according to the situation and it also enhance the efficiency of the dynamic clustering. Experimental results show that new proposed technique is more efficient, reliable and provide more throughput as compare to the existing technique.
\end{abstract}

Keywords: Learning, Neural Networks Clusters, Boltzmann learning.

\section{INTRODUCTION}

A wireless sensor network (WSN) is a collection of electronic terminals or nodes distributed over a geographical area to be monitored. Each node in the network performs the function of sensing, transmitting and receiving the desired information. Every node typically carries a transceiver, a processing unit and a power source i.e. a battery to perform basic operations of a network.[1,2]

WSN is a special type of ad-hoc network in which node density is much greater than traditional ad-hoc networks. The nodes in the network are usually static and are energy constrained and works in a collective manner to collect data and then transmit it to the central processing system called a base station or a sink, where it is processed further.[3,4]. Sensor nodes are energy-constrained devices and the energy consumption is generally associated with the amount of gathered data, since communication is often the most expensive activity in terms of energy. For that reason, algorithms and protocols designed for WSNs should consider the energy consumption in their conception [5],[6], [7], [8]. Various algorithms have been proposed to provide data aggregation during the routing in WSNs. Some of them are tree-based algorithms, clusterbased algorithms while others are simply structure-less algorithms [9]. In [10] short path tree algorithm is discussed which is tree-based routing approach and it has static routes. In [11] Information fusion based role assignment algorithm is discussed. The InFRA algorithm aims at building the shortest path tree that maximizes information fusion. Low Energy Adaptive Clustering Hierarchy (LEACH) proposed in [12] is one of the most famous WSN hierarchical routing algorithms. In LEACH, the nodes organize themselves into local cluster, the protocol is divided into a setup phase when the clusters are organized and a steady-state phase when data is transferred from the nodes to the cluster head and on to the sink. In [13] Data Routing For In-Network Aggregation (DRINA) Approach is proposed which is a cluster based approach. The Cluster head $(\mathrm{CH})$ acts as the aggregation point. It aims at maximizing the overlapping routes for data transfer and reducing the communication cost. The drawbacks of DRINA are: the routes are static, the overhead is high and the link failure.

In this paper we propose an algorithm based on DRINA to minimize the link failure. The main aim of the proposed algorithm is to minimize the energy consumption of the network. This algorithm also takes into account that if one node fails due to any reason, communication is still maintained.

The rest of the paper is organised as follows: Section II provides the brief idea about DRINA. Section III describes the proposed algorithm. Simulation and results are given in section IV and finally section $\mathrm{V}$ gives the conclusion of the paper.

\section{DRINA}

Data Routing for In-Network Aggregation (DRINA) Approach: The main goal of the DRINA algorithm is to build a routing tree with the shortest paths that connect all source nodes to the sink while maximizing data aggregation. This algorithm considers the following roles in the routing infrastructure creation:

1. Collaborator: A node that detects an event and reports the gathered data to a coordinator node. 
2. Coordinator: A node that also detects an event and is input: $\rightarrow$ number of nodes, number of rounds responsible for gathering all the gathered data sent by output: $\rightarrow$ packets transmitted to Base Station collaborator nodes, aggregating them and sending the * creating heterogeneity in node energies* result toward the sink node.

3. for $\mathrm{I} \leftarrow 1$ to rounds

3. Sink: A node interested in receiving data from a set of $\mathbf{4}$. for $\mathrm{j} \leftarrow 1$ to $\mathrm{N}$ coordinator and collaborator nodes.

* compute probability of distribution of normal nodes 4. Relay: A node that forwards data toward the sink. The DRINA algorithm can be divided into three stages.

In Stage 1, the hop tree from the sensor nodes to the sink node is built. In this phase, the sink node starts building the hop tree that will be used by Coordinators for data forwarding purposes. Stage 2 consists of cluster formation and cluster-head election among the nodes that detected the occurrence of a new event in the network. Finally, Stage 3 is responsible for both setting up a new route for the reliable delivering of packets and updating the hop tree.

\section{PROPOSED ALGORITHM}

\section{A. EDRINA}

Enhanced Data Routing For In-Network Aggregation (EDRINA) is a cluster based approach, which mainly aims at improving the performance in the network while maximizing the data aggregation in the network. Here, $\mathrm{CH}$ acts as the aggregation point. It makes use of Periodic Simple Aggregation as its timing strategy for data aggregation. It requires each node to wait for a predefined period of time while aggregating all received data packet and then forward a single packet with the result of the aggregation.

First of all, nodes are deployed in the network. After that cluster heads are chosen according to the bully algorithm. The nodes in a cluster having maximum energy are selected a cluster head. All the communication from nodes to sink is done through cluster heads. The path which is established for communication is known as predefined path. In DRINA protocol when data transfer to sink through cluster heads, sometimes one cluster heads fails due to battery degradation. Link failure problem occur due to battery degrade. The data loss begins and further communication stops. To overcome this problem a new technique will be proposed based upon relay nodes. In this work, relay nodes are added externally in the clusters. These nodes have the more battery power as compared to other nodes. After that cluster head ask each node of the cluster in the predefined path for its battery power. The node which has highest battery power will be chosen as cluster head. Now this new cluster head will participate in the communication and solve link failure problem in the predefined path of the network.

\section{B. Algorithm}

*Setup Phase *

1. START

* In Setup Phase the tasks are performed and initiated by Sink *

\section{INITIALIZATION}

$\mathrm{k}$ is desired number of clusters set by $\mathrm{p}_{\mathrm{opt}} \mathrm{N}$, where $\mathrm{N}$ is the number of sensor nodes deployed from these we take fraction of nodes $\mathrm{m}, \mathrm{m}_{0}, \mathrm{~m}_{1}$ and $\mathrm{m}_{2}$ to have $\mathrm{a}, \mathrm{b}, \mathrm{u}$ and $\mathrm{mm}$ times more energy than normal nodes respectively.

\section{from all nodes*}

\section{5. $\mathrm{J} \leftarrow 1$ to $\mathrm{N}$}

*compute probability of distribution of advance nodes from normal nodes*

6. $\mathrm{J}(\mathrm{A}) \leftarrow 1$ to $\mathrm{J}$

* compute probability of distribution of super nodes from advanced nodes*

7. $\mathrm{J}(\mathrm{S}) \leftarrow 1$ to $\mathrm{J}(\mathrm{A})$

*compute probability of distribution of super-ultra nodes from super nodes*

8. $\mathrm{J}(\mathrm{US}) \leftarrow 1$ to $\mathrm{J}(\mathrm{S})$

*compute probability of distribution of extreme nodes from super-ultra nodes*

9. $\mathrm{J}(\mathrm{E}) \leftarrow 1$ to $\mathrm{J}(\mathrm{US})$

*Select Cluster Heads $\mathrm{CH}_{\mathrm{i}}$ from $\mathrm{N}$ nodes based on $\mathrm{p}_{\mathrm{i}}$ i.e. average probability of a node to be Cluster Head*

10. $\mathrm{T}(\mathrm{N}) \rightarrow \mathrm{p}_{\mathrm{i}} \quad *$ Probability Threshold*

11. Create TDMA Schedule for all Nodes of Cluster

* End of Setup Phase *

12. for $v \leftarrow 1$ to round

$*$ round $\rightarrow$ Total number of rounds *

* Responsible Node Selection Phase *

13. for $\mathrm{i} \leftarrow 1$ to $\mathrm{k}$

14. for Alive_node $\leftarrow 1$ to $s_{i}$

*Alive_node $\rightarrow$ Nodes whose residual energy is greater than or equal to threshold energy $\left(\mathbf{E}_{\text {trsh }}\right)^{*}$

15. Send $\left(\mathrm{E}_{\text {avg }}{ }_{\mathrm{i}}^{\mathrm{i}}, \mathrm{E}_{\text {residual }} \mathrm{i}\right)$ information to $\mathrm{CH}_{\mathrm{i}}$

* $\mathrm{CH}_{\mathrm{i}}$ performs following tasks *

16. Call Responsible_Node_Selection ( )

* Select CHi for the current round and TCHi for next round *

*End for phase*

Steady State Phase

17. For i $\leftarrow 1$ to $\mathrm{k}$

18. For Alive_node $\leftarrow 1$ to $s_{i}$

19. Send data to $\mathbf{C H}_{\mathbf{i}}$

* Data transmission by Alive_nodes *

20. Send aggregated data to Sink/Base station

* Data transmission by $\mathrm{CH}_{\mathrm{i}}$ *

* End of the phase*

\section{SIMULATION AND RESULTS}

\section{Simulation Parameters}

\begin{tabular}{|l|l|}
\hline Simulation Parameter & Value \\
\hline Simulator & Matlab \\
\hline Area & $100 * 100 \mathrm{~m}$ \\
\hline No. of nodes & 100 \\
\hline No. of rounds & 5000 \\
\hline Initial energy of each node & 0.1 joule \\
\hline ETX & $50 * 0.000000001$ \\
\hline ERX & $50 * 0.000000001$ \\
\hline
\end{tabular}

The whole scenario is implemented in MATLAB. 


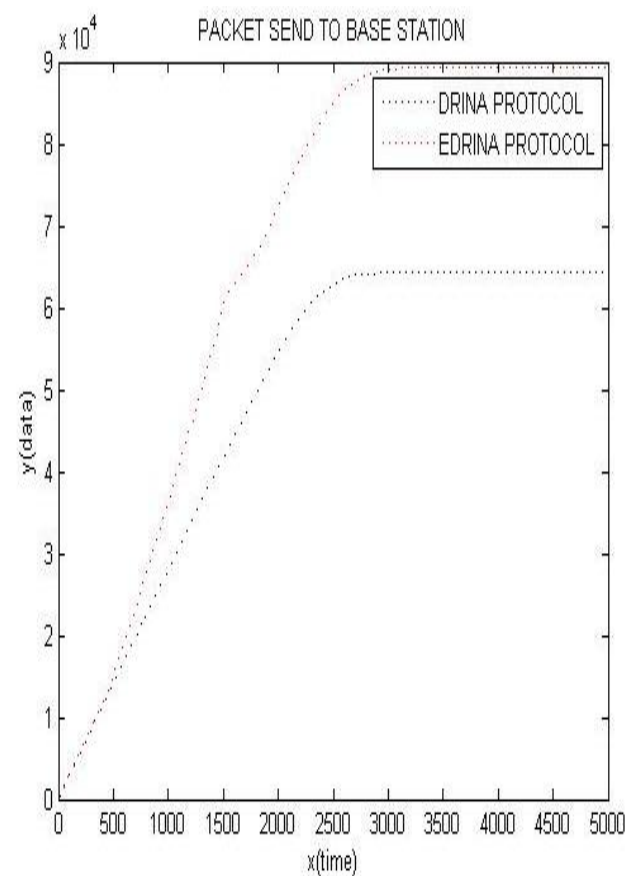

Fig. 4.1: Comparison of network lifetime

As shown in figure 4.1, The network deployed with finite number 100 nodes and data is transmitted in 5000 rounds. In the existing algorithm $6.9 * 10^{\wedge} 4$ number of packets are transmitted to base station and in enhanced algorithm total number of $8.9 * 10^{\wedge} 4$ number of packets are transmitted to base station.

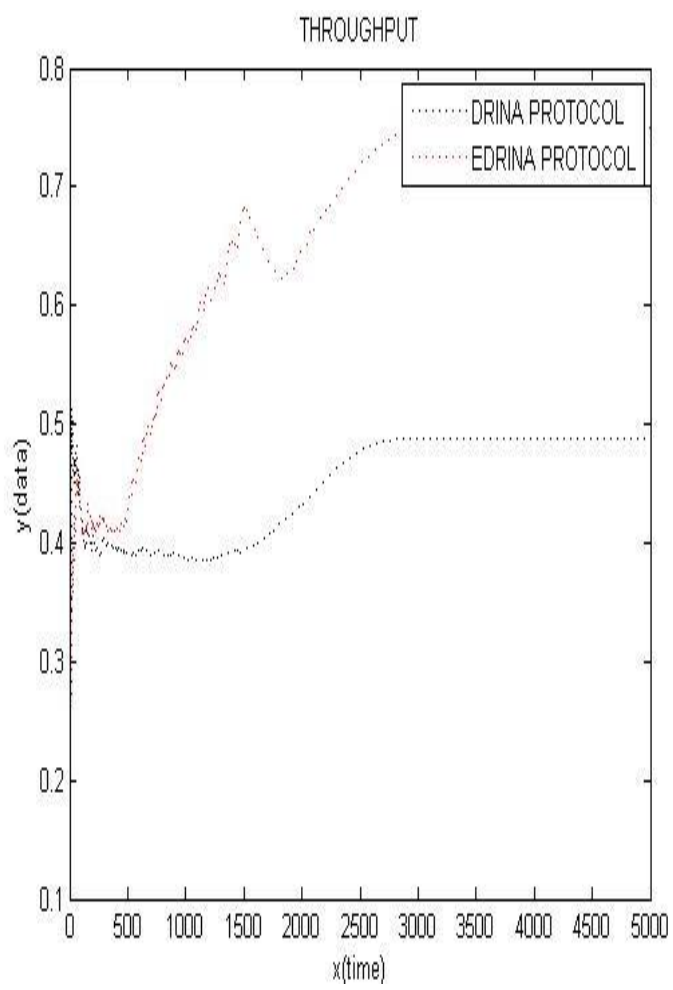

Fig.4.2 Throughput Graph

As shown in figure 4.2, the total number of 5000 rounds is required to transmit data to base station. In the existing algorithm less number of packets is transmitted to base station as compared enhanced algorithm. As shown in figure, the throughput of enhanced algorithm is more as compare to existing algorithm.

\section{CONCLUSION}

Wireless Sensor nodes are very small in size and have limited resources. In far places it is very difficult to recharge or replace the battery of the sensor nodes. In such conditions, we have focused to reduce the battery consumption of the sensor nodes. In DRINA, there is a problem of link failure which degrades the performance of the network. In this paper we had proposed a technique in which relay nodes were added and participated in communication to reduce link failure problem in terms of throughput and network lifetime. Performance of DRINA can be further improved by using efficient data aggregation techniques.

\section{REFERENCES}

[1] I.F. Akyildiz, W. Su, Y. Sankarasubramaniam, E. Cayirci, "Wireless sensor networks: a survey," 12 December 2001.

[2] S. M. Lasassmeh, J. M. Conrad, "Time Synchronization in Wireless Sensor Networks: A Survey," IEEE SoutheastCon Proceedings, pp. 242 - 245, March 2010.

[3] B. Hofmann-Wellenhof, H. Lichtenegger, and J. Collins, Global Positioning System: Theory and Practice, Springer-Verlag, Fifth edition, 2001

[4] T. Jain, "Wireless Environmental Monitoring System Using Data Aggregation in a Bidirectional Hybrid Protocol," Information Systems, Technology and Management Communications in Computer and Information Science, vol. 285, pp. 414-420, 2012.

[5] S. Olariu, Q. Xu, and A. Zomaya, "An Energy-Efficient SelfOrganization Protocol for Wireless Sensor Networks," Proc. IEEE Intelligent Sensors, Sensor Networks and Information Processing Conf. (ISSNIP), pp. 55-60, Dec. 2004.

[6] H.S. AbdelSalam and S. Olariu, "A Lightweight Skeleton Construction Algorithm for Self-Organizing Sensor Networks," Proc. IEEE Int'l Conf. Comm. (ICC), pp. 1-5, http://dblp.uni-trier. de/db/conf/icc/icc2009.html\#AbdelSalamO09, 2009.

[7] L. Villas, A. Boukerche, R.B. de Araujo, and A.A.F. Loureiro, "Highly Dynamic Routing Protocol for Data Aggregation in Sensor Networks," Proc. IEEE Symp. Computers and Comm. (ISCC), pp. 496-502, http://dx.doi.org/10.1109/ISCC.2010.5546580, 2010.

[8] L.A. Villas, A. Boukerche, H.A. de Oliveira, R.B. de Araujo, and A.A. Loureiro, "A Spatial Correlation Aware Algorithm to Perform Efficient Data Collection in Wireless Sensor Networks," Ad Hoc Networks, http://www.sciencedirect.com/science/article/pii/S157 $0870511001892,2011$.

[9] K. Romer and F. Mattern, "The Design Space of Wireless Sensor Networks," IEEE Wireless Comm., vol. 11, no. 6, pp. 54-61, Dec. 2004.

[10] B. Krishnamachari, D. Estrin, and S.B. Wicker, "The Impact of Data Aggregation in Wireless Sensor Networks," Proc. 22nd Int'l Conf. Distributed Computing Systems (ICDCSW '02), pp. 575578,2002

[11] E.F. Nakamura, H.A.B.F. de Oliveira, L.F. Pontello, and A.A.F. Loureiro, "On Demand Role Assignment for Event-Detection inSensor Networks," Proc. IEEE 11th Symp. Computers and Comm. (ISCC '06), pp. 941-947, 2006.

[12] Marc Lee and Vincent W.S Wong, "An Energy Aware Spanning Tree Algorithm For Data Aggregation in Wireless Sensor Networks," IEEE 2005.

[13] Villas L.A, Boukerche A, Ramos H.S, De and Loureiro A.A.F (2013)"DRINA: A Lightweight Aggregation in Wireless Sensor Networks," IEEETrans.,on computers, vol.62 No.4, pp 676689.,2013. 